\title{
On the use of photometric redshifts for X-ray selected AGNs ${ }^{\star}$
}

\author{
S. Kitsionas ${ }^{1,2}$, E. Hatziminaoglou ${ }^{3}$, A. Georgakakis ${ }^{1}$, and I. Georgantopoulos ${ }^{1}$ \\ 1 Institute of Astronomy \& Astrophysics, National Observatory of Athens, I. Metaxa and V. Pavlou str., \\ P. Penteli, 15236 Athens, Greece \\ 2 Astrophysikalisches Institut Potsdam, An der Sternwarte 16, 14482 Potsdam, Germany \\ e-mail: skitsionas@aip.de \\ 3 Instituto de Astrofisica de Canarias, C/ Vía Làctea, s/n, 38205 La Laguna, Tenerife, Spain
}

Received 27 August 2004 / Accepted 3 December 2004

\begin{abstract}
In this paper we present photometric redshift estimates for a sample of X-ray selected sources detected in the wide-field $\left(\sim 2 \mathrm{deg}^{2}\right)$, bright $\left[f_{\mathrm{X}}(0.5-8 \mathrm{keV}) \approx 10^{-14} \mathrm{erg} \mathrm{s}^{-1} \mathrm{~cm}^{-2}\right] \mathrm{XMM}-N e w t o n / 2 \mathrm{dF}$ survey. Unlike deeper X-ray samples comprising a large fraction of sources with colours dominated by the host galaxy, our bright survey primarily probes the QSO X-ray population. Therefore photometric redshift methods employing both galaxy and QSO templates need to be used. We employ the photometric redshift technique of Hatziminaoglou et al. (2000) using 5-band photometry from the SDSS. We separate our X-ray sources according to their optical profiles into point-like and extended. We apply QSO and galaxy templates to the point-like and extended sources respectively. X-ray sources associated with Galactic stars are identified and discarded from our sample on the basis of their unresolved optical light profile, their low X-ray-to-optical flux ratio and their broad-band colours that are best fit by stellar templates. Comparison of our results with spectroscopic redshifts available allows calibration of our method and estimation of the photometric redshift accuracy. For $\sim 70$ per cent of the point-like sources photometric redshifts are correct within $\delta z \lesssim 0.3$ (or $\sim 75$ per cent have $\delta z /(1+z) \lesssim 0.2$ ), and the rms scatter is estimated to be $\sigma_{z}=0.30$. Also, in our $X$-ray selected point-like sample we find that about 7 per cent of the sources have optical colours redder than those of optically selected QSOs. Photometric redshifts for these systems using existing QSO templates are most likely problematic. For the optically extended objects the photometric redshifts work only in the case of red $(g-r>0.5 \mathrm{mag})$ sources yielding $\delta z \lessgtr 0.15$ and $\delta z /(1+z) \lessgtr 0.2$ for 73 and 93 per cent respectively. The results above are consistent with earlier findings from the application of combined galaxy/QSO photometric redshift techniques in the Chandra Deep Field North. However, we find that the above photometric redshift technique does not work in the case of extended sources with blue colours $(g-r<0.5)$. Although these form a significant fraction of the extended sources ( $\approx 40 \%)$, they cannot be fit successfully by QSO or galaxy templates, or any linear combination of the two.
\end{abstract}

Key words. techniques: photometric - quasars: general - galaxies: active - galaxies: distances and redshifts - X-rays: galaxies

\section{Introduction}

In the past few years, X-ray surveys carried out by the XMM-Newton and the Chandra observatories have significantly improved our understanding of X-ray selected AGNs (Hasinger et al. 2001; Brandt et al. 2001; Giacconi et al. 2001; Barger et al. 2002; Brandt et al. 2002; Mainieri et al. 2002; Georgantopoulos et al. 2004). Moreover, serendipitous studies using the large volume of archival data provided by these missions are well underway, aiming to further push our knowledge of both the X-ray properties and the evolution of AGNs (Green et al. 2004; Kim et al. 2004a, 2004b).

* Full Tables 1 and 2 are only available in electronic form at the CDS via anonymous ftp to cdsarc.u-strasbg.fr $(130.79 .128 .5)$ or via

http://cdsweb.u-strasbg.fr/cgi-bin/qcat?J/A+A/434/475
To maximise their scientific impact, the above largescale projects require redshift information for a large number of X-ray sources. For example the recently released XMM-Newton Serendipitous Source Catalogue comprises about 50000 sources, with more archival observations being accumulated. Spectroscopic follow-up programs are underway (e.g. Barcons et al. 2002) but they are expensive in telescope time and completion may take years. Moreover, many sources are expected to be optically faint, rendering spectroscopic observations difficult even with $10-\mathrm{m}$ class telescopes.

The issues above have motivated the development of photometric redshift techniques that use multiwaveband imaging to estimate the redshifts of extragalactic sources. These methods have become increasingly popular over the last few years because of their effectiveness and the relatively small investment in observing time that they require compared to spectroscopy. 
For normal galaxies in particular, with optical colours dominated by stellar processes, photometric redshifts have proven exceptionally successful, achieving accuracies that allow detailed statistical studies (e.g. Budavari et al. 2003; Csabai et al. 2003).

For AGN-dominated systems with featureless continua, photometric redshift estimates are more challenging. Nevertheless, significant effort has been expended in this direction in recent years. Budavari et al. (2001) and Richards et al. (2001) used Sloan Digital Sky Survey (SDSS) data to estimate photometric redshifts of optically selected QSOs; their results are accurate within $\delta z<0.2$ for 70 per cent of their sample.

For $X$-ray selected samples, Gonzalez \& Maccarone (2002) have estimated photometric redshifts for X-ray sources to the limit $f_{\mathrm{X}}(0.5-8 \mathrm{keV}) \approx 10^{-16} \mathrm{erg} \mathrm{s}^{-1} \mathrm{~cm}^{-2}$. Using galaxy templates these authors find good agreement between photometric and spectroscopic redshifts only for systems dominated by light from the host galaxy rather than the central engine. This class of sources is the dominant population, representing about 90 per cent of their X-ray selected sample to the flux limit above. Similarly, Mobasher et al. (2004) used optical data from the GOODS survey to estimate photometric redshifts for X-ray selected sources in the Chandra Deep Field North $\left[f_{\mathrm{X}}(0.5-8 \mathrm{keV}) \approx 10^{-16} \mathrm{erg} \mathrm{s}^{-1} \mathrm{~cm}^{-2}\right]$. Although for most of these X-ray faint sources galaxy templates provide adequate photometric redshift estimates, they fail for powerful QSOs. Babbedge et al. (2004) recently presented a combined galaxyQSO template approach to derive photometric redshifts for the $\mathrm{X}$-ray sources in the Chandra Deep Field North (CDFN).

The use of combined galaxy-QSO templates is imperative in the case of X-ray surveys at brighter limits, which comprise a significant fraction of powerful AGNs with optical light dominated by the central engine. In this paper we address this issue, providing photometric redshifts for X-ray sources detected in a wide-field $\left(\sim 2 \mathrm{deg}^{2}\right)$, shallow $\left[f_{\mathrm{X}}(0.5-8 \mathrm{keV}) \approx\right.$ $10^{-14} \mathrm{erg} \mathrm{s}^{-1} \mathrm{~cm}^{-2}$ ] XMM-Newton survey near the North Galactic Pole region (part of the XMM-Newton/2dF survey; Georgakakis et al. 2003, 2004). The strength of this sample is that it overlaps with the SDSS (York et al. 2000) and hence, high quality 5-band photometry is available, allowing photometric redshift estimates. Also spectroscopic redshifts are available for a sub-sample of the X-ray source population from the 2dF QSO Redshift Survey (2QZ; Croom et al. 2001), the SDSS survey (York et al. 2000; Stoughton et al. 2002) as well as our own spectroscopic campaign. The spectroscopic redshifts allow both calibration of the photometric redshift method and estimation of its accuracy.

In the following section we give a detailed description of both the X-ray and optical (photometric and spectroscopic) data used in the present study. In Sect. 3 we give a brief summary of the photometric redshift method while Sect. 4 presents our results. These are discussed in Sect. 5. Our conclusions are summarised in Sect. 6.

\section{The sample}

The X-ray data are from the XMM-Newton/2dF survey, a widearea, shallow $\left[f_{\mathrm{X}}(0.5-8 \mathrm{keV}) \approx 10^{-14} \mathrm{erg} \mathrm{s}^{-1} \mathrm{~cm}^{-2}\right] \mathrm{X}$-ray sample near the North and the South Galactic Pole regions. The X-ray data reduction, source extraction, flux estimation and catalogue generation have been described in detail by Georgakakis et al. (2003, 2004).

In the present study we concentrate on the XMM-Newton/2dF survey sub-sample near the North Galactic Pole region. This is because of the wealth of homogeneous follow-up optical photometric and spectroscopic observations available in this region. A total of 291 X-ray sources have been detected in the $0.5-8 \mathrm{keV}$ spectral band above the $5 \sigma$ threshold.

The optical photometric data are from the SDSS Early Data Release (EDR; Stoughton et al. 2002). The SDSS is an ongoing imaging and spectroscopic survey that aims to cover about $10000 \mathrm{deg}^{2}$ of the sky. Photometry is performed in 5 bands (ugriz; Fukugita et al. 1996) to a limiting magnitude of $g \approx 23.0$ mag. These data are used for the optical identification of the X-ray sample using the method described by Downes et al. (1986). We propose optical counterparts for 193 out of 291 X-ray sources.

Spectroscopic data for the optically brighter X-ray sources are from the SDSS and the 2QZ. The SDSS will obtain spectra for over 1 million objects, including galaxies brighter than $r=17.7 \mathrm{mag}$, luminous red galaxies to $z=0.45$ and colour selected QSOs (York et al. 2000; Stoughton et al. 2002; Richards et al. 2002). The $2 \mathrm{QZ}$ is a large-scale spectroscopic campaign that fully exploits the capabilities of the $2 \mathrm{dF}$ multifibre spectrograph on the $4 \mathrm{~m}$ Anglo-Australian Telescope (AAT). This project provides high quality spectra, redshifts and spectral classifications for 23000 optically selected $b_{j}<20.85$ mag QSOs (Croom et al. 2001). In addition to publicly available spectroscopic data from the 2QZ and the SDSS spectroscopic surveys, we use redshift measurements from our own on-going spectroscopic campaign of the XMM-Newton/2dF sources. Part of these data is presented by Georgakakis et al. (2004).

\section{The photometric redshift method}

Photometric redshifts are estimated using the method described by Hatziminaoglou et al. (2000; HMP00). In brief, this is based on a standard $\chi^{2}$ minimisation technique where the photometric redshift of an object is estimated by comparing its multiband photometry with model QSO Spectral Energy Distribution (SED) templates, shifted in redshift space and integrated through the bandpass throughput functions. The redshift is allowed to vary in the range $0-6$ with a linear step of 0.1 . For each template and at each redshift, the $\chi^{2}$ probability of each source is calculated. Once a local minimum in $\chi^{2}$ is found, a refined search for a better solution is made around it, with a step of 0.01. More than one local minimum might exist, as in all template fitting techniques. The photometric redshift of the source is then given as the redshift corresponding to the global minimum of these $\chi^{2}$ values.

The QSO templates are produced by varying the optical power-law spectral index of simulated QSO spectra between 0 and 1, while keeping the UV spectral index constant at 1.76 (Wang et al. 1998). We note, however, that varying the UV spectral index does not modify our results and conclusions. 
Emission lines (Ly $\alpha, \operatorname{Ly} \beta, \mathrm{CIV},[\mathrm{CIII}] 1909, \mathrm{MgII}, \operatorname{SiIV}, \mathrm{H} \alpha$, $\mathrm{H} \beta$ and $\mathrm{H} \gamma)$ as well as the blue bump centred at $3000 \AA$ are also included in the QSO-template SEDs. The Ly $\alpha$ forest has been modelled following Madau (1995). No reddening has been applied to the QSO-template SEDs.

Using the spectroscopic data available for our sample, we attempt to fine-tune the spectral line properties (i.e. equivalent widths and relative intensities) of the QSO-template SEDs. This is in order to calibrate the HMP00 method on $\mathrm{X}$-ray selected samples. We conclude that the spectral line profiles used by HMP00 are also optimal for our X-ray selected AGN sample.

The template SED library of the HMP00 technique also comprises stellar spectra for a range of spectral types including white dwarfs (Pickles 1998). These are used to identify candidate stars (Hatziminaoglou et al. 2002) within our X-ray selected sample. The observed mean spectra of four different galaxy types (E/S0, Sbc, Scd, Im) from Coleman et al. (1980; CWW) are also used in this paper to estimate photometric redshifts for X-ray sources dominated by light from the host galaxy.

For the photometric redshift estimation we employ the SDSS 5-band photometry using PSF magnitudes (psfMag SDSS parameter) for optically unresolved sources and Petrosian magnitudes (petroMag SDSS parameter) for optically extended systems.

\section{Results}

\subsection{The spectroscopic sub-sample}

We apply the method described in the section above to the subsample of X-ray sources with spectroscopic data available to assess the success rate of our technique. The spectroscopic subsample comprises 71 optically unresolved (e.g. point-like morphology) systems and 26 sources with extended optical light profile. The morphological classification has been obtained from the SDSS. This is reliable at the 95 per cent confidence level at the magnitude limit $r=21 \mathrm{mag}$ (York et al. 2000; Stoughton et al. 2002). At fainter magnitudes the star/galaxy classification becomes less robust.

We treat extended and point-like sources separately using galaxy and QSO templates respectively. X-ray sources with unresolved optical light profile are most likely associated with distant powerful AGNs where the light from the central engine dominates the optical emission. QSO-template SEDs are clearly more appropriate for these sources. The X-ray sources with extended optical light profile are most likely relatively nearby galaxies where the central source does not dominate their optical light. Therefore, galaxy templates are likely appropriate for these systems.

Before applying the HMP00 method to the full spectroscopic catalogue we identify and exclude from our analysis Galactic star candidates. In particular, we first identify optically unresolved (e.g. point-like morphology) sources with low X-ray-to-optical flux ratio $\left(\log f_{\mathrm{X}} / f_{\text {opt }}<-1\right.$; total of 13), i.e. in the region of the parameter space occupied by Galactic stars (Stocke et al. 1991). Secondly, for these

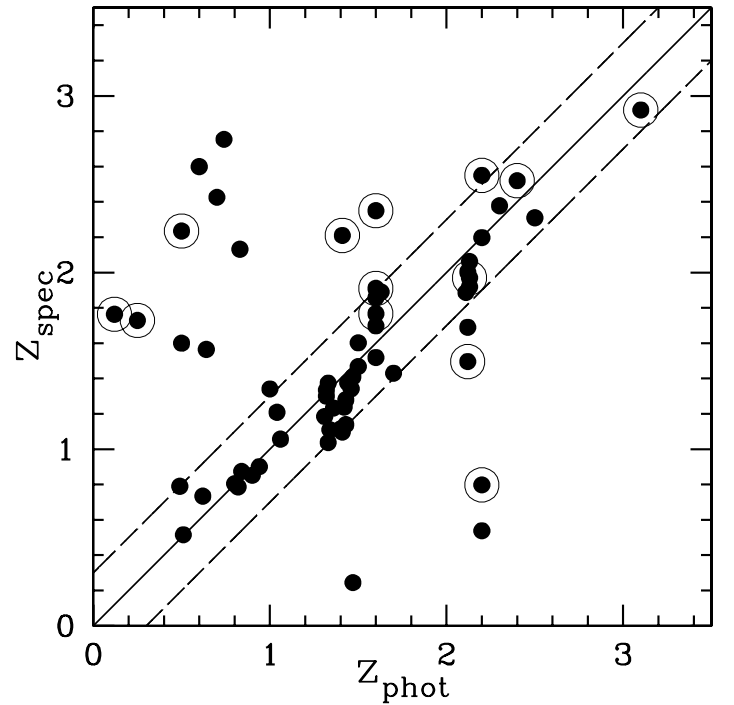

Fig. 1. Comparison between $z_{\text {spec }}$ and $z_{\text {phot }}$ for X-ray sources with point-like optical light profile and spectroscopic redshift measurements. A large circle on top of a symbol is for sources with $r>21$. The solid line is the $z_{\text {spec }}=z_{\text {phot }}$ locus. The dashed lines define the $\delta z= \pm 0.3$ envelope around the $z_{\text {spec }}=z_{\text {phot }}$ line.

$\log f_{\mathrm{X}} / f_{\text {opt }}<-1$ sources we apply the HMP00 technique using both QSO and stellar templates in an attempt to identify objects with optical SEDs consistent with those of stars. This is because the $\log f_{\mathrm{X}} / f_{\mathrm{opt}}<-1$ regime also comprises a small fraction of QSOs in addition to other classes of sources. Of the 13 sources with $\log f_{\mathrm{X}} / f_{\text {opt }}<-1$ a total of 9 are best fit by stellar templates. All of them are spectroscopically identified Galactic stars (Georgakakis et al. 2004). The remaining 4 sources are best fit by QSO templates. This classification is consistent with the optical spectroscopy available for these objects (Georgakakis et al. 2004). Therefore, our method based on both X-ray and optical broad-band information is successful in identifying all the Galactic stars within the spectroscopic sub-sample. In the remaining of this paper we shall not consider the 9 Galactic stars identified in our spectroscopic sub-sample.

For the remaining 62 optically unresolved X-ray sources we have applied the photometric redshift technique of HMP0O using QSO templates only. The results are presented in Table 1 and are compared with the spectroscopic redshift measurements in Fig. 1. A total of 42 out of 62 objects ( $\approx 68$ per cent) have photometric redshift estimates correct within $\delta z \lesssim 0.3$. For this sample we estimate a fractional mean error defined as

$\overline{\delta z /(1+z)}=\sum\left(\frac{z_{\text {phot }}-z_{\text {spec }}}{1+z_{\text {spec }}}\right) / N$,

$\overline{\delta z /(1+z)}=-0.02$, and an rms scatter defined as

$\sigma_{z}^{2}=\sum\left(\frac{z_{\text {phot }}-z_{\text {spec }}}{1+z_{\text {spec }}}\right)^{2} / N$

$\sigma_{z}=0.30$. The agreement between photometric and spectroscopic redshifts is fair out to $z \approx 1.5$ and becomes poorer at higher redshifts. As will be discussed in the next section, these problematic redshift estimates are likely because of strong emission lines falling between the SDSS filters. As already 
Table 1. Photometric redshifts for the $88 \mathrm{X}$-ray sources with spectroscopic identification in the XMM-Newton/2dF sample.

\begin{tabular}{|c|c|c|c|c|c|c|c|c|c|c|c|c|}
\hline \multirow[t]{2}{*}{$\overline{~ \# ~}$} & \multicolumn{3}{|c|}{$\overline{\alpha_{\text {opt }}(\mathrm{J} 2000)}$} & \multicolumn{3}{|c|}{$\bar{~} \delta_{\text {opt }}(\mathrm{J} 2000)$} & \multirow[t]{2}{*}{$z_{\text {phot }}$} & \multirow[t]{2}{*}{$z_{\text {spec }}$} & \multirow[t]{2}{*}{ Survey $^{1}$} & \multirow[t]{2}{*}{$\overline{r^{2}}$} & \multirow[t]{2}{*}{$\overline{g-r^{2}}$} & \multirow[t]{2}{*}{ Morph $^{2}$} \\
\hline & $\mathrm{h}$ & $\mathrm{m}$ & $\mathrm{s}$ & $\mathrm{o}$ & ' & " & & & & & & \\
\hline 1 & 13 & 40 & 41.43 & -00 & 17 & 26.52 & 0.740 & 2.754 & 2 & 20.108 & 0.129 & $\mathrm{P}$ \\
\hline 2 & 13 & 40 & 49.98 & 00 & 26 & 44.84 & 2.120 & 1.496 & 4 & 21.368 & 0.057 & $\mathrm{P}$ \\
\hline 3 & 13 & 40 & 50.18 & 00 & 07 & 28.09 & 0.940 & 0.901 & 4 & 19.761 & 0.183 & $\mathrm{P}$ \\
\hline 4 & 13 & 40 & 59.07 & 00 & 05 & 04.63 & 0.600 & 2.599 & 1 & 18.885 & 0.018 & $\mathrm{P}$ \\
\hline 5 & 13 & 40 & 59.24 & -00 & 19 & 44.94 & 1.600 & 1.857 & 1 & 19.956 & 0.086 & $\mathrm{P}$ \\
\hline 6 & 13 & 41 & 01.35 & -00 & 27 & 32.15 & 1.000 & 0.619 & 4 & 20.024 & 0.298 & E \\
\hline 7 & 13 & 41 & 02.92 & 00 & 15 & 47.20 & 1.330 & 1.038 & 4 & 19.628 & 0.253 & $\mathrm{P}$ \\
\hline 8 & 13 & 41 & 03.22 & -00 & 24 & 48.71 & 0.640 & 1.565 & 4 & 20.644 & 0.054 & $\mathrm{P}$ \\
\hline 9 & 13 & 41 & 07.90 & -00 & 17 & 25.37 & 2.200 & 0.798 & 4 & 21.324 & 0.110 & $\mathrm{P}$ \\
\hline 10 & 13 & 41 & 16.96 & 00 & 21 & 50.40 & 1.900 & 0.721 & 4 & 21.172 & -0.087 & E \\
\hline 79 & 13 & 44 & 52.91 & 00 & 05 & 20.26 & 0.100 & 0.087 & 1 & 16.325 & 0.603 & E \\
\hline 80 & 13 & 44 & 54.64 & -00 & 19 & 07.57 & 0.900 & 0.852 & 2 & 20.397 & 0.116 & $\mathrm{P}$ \\
\hline 81 & 13 & 44 & 55.82 & -00 & 01 & 16.46 & 2.200 & 2.198 & 2 & 20.893 & 0.049 & $\mathrm{P}$ \\
\hline 82 & 13 & 44 & 58.26 & 00 & 16 & 22.87 & 0.100 & 0.145 & 4 & 18.022 & 0.587 & E \\
\hline 83 & 13 & 44 & 58.26 & -00 & 35 & 57.59 & 0.100 & 0.465 & 4 & 19.389 & 0.904 & E \\
\hline 84 & 13 & 44 & 59.45 & -00 & 15 & 59.54 & 1.470 & 0.245 & 1 & 17.529 & 0.074 & $\mathrm{P}$ \\
\hline 85 & 13 & 45 & 08.04 & -00 & 05 & 26.70 & 2.500 & 0.730 & 4 & 19.776 & 0.179 & E \\
\hline 86 & 13 & 45 & 12.30 & -00 & 31 & 31.08 & 2.120 & 1.690 & 2 & 20.521 & -0.068 & $\mathrm{P}$ \\
\hline 87 & 13 & 45 & 15.31 & 00 & 15 & 22.54 & 0.100 & 0.089 & 1 & 16.123 & 0.909 & E \\
\hline 88 & 13 & 45 & 19.09 & 00 & 31 & 00.44 & 0.500 & 0.730 & 4 & 19.511 & 0.721 & $\mathrm{E}$ \\
\hline
\end{tabular}

${ }^{1}$ Spectroscopy from: 1 - SDSS; 2 - 2QZ; 3 - AAT with AUTOFIB or LDSS (Georgantopoulos et al. 1996); 4 - our own campaign (Georgakakis et al. 2004).

${ }^{2} r$ : SDSS $r$ band magnitude; $g-r$ : SDSS $g-r$ colour in mag; morph: SDSS optical morphology (P - point-like; E - extended).

discussed above, the star-galaxy separation is 95 per cent reliable at $r=21 \mathrm{mag}$ and degrades at fainter magnitudes. This suggests that extended sources with $r>21$ mag may be erroneously classified point-like and vice versa resulting in the use of wrong templates to determine their redshift. In the optically point-like sub-sample there are 13 sources with $r>21 \mathrm{mag}$ (marked with a large circle in Fig. 1). As can be seen in Fig. 1, about half of them have $\delta z>0.3$. Using galaxy instead of QSO templates does not improve their photometric redshift estimates. The low success rate for these systems is most likely because of larger photometric errors than in optically brighter sources.

For the 26 optically extended X-ray sources in the $\mathrm{XMM}-$ Newton/2dF sample with spectroscopic data we have applied the HMP00 technique using galaxy SED templates only. The photometric redshift estimates for these sources are also presented in Table 1. Figure 2 plots $\delta z$ as a function of $g-r$ colour for the spectroscopic sub-sample of extended sources (total of 26 sources), while Fig. 3 plots spectroscopic against photometric redshift for the same sources. It is clear from these figures that for sources with red optical colours $(g-r>0.5 \mathrm{mag})$ our method performs well with a mean error of $\overline{\delta z /(1+z)}=-0.01$ and rms scatter $\sigma_{z}=0.10$. For objects with blue colours $(g-r<0.5 \mathrm{mag})$ however, our method is not as successful, giving $\overline{\delta z /(1+z)}=+0.59$ and $\sigma_{z}=0.99$.
The photometric redshifts of this blue X-ray source population cannot be improved by using QSO or starburst templates, suggesting that their optical light is a mix of both AGN and stellar emission.

We further explore this possibility in an attempt to improve the photometric redshift estimates for these sources by linearly combining QSO (total of 3; see Sect. 3) and galaxy (total of 4 from CWW) templates. These are normalised at $5000 \AA$, although the choice of the normalisation wavelength does not alter our results. For each of the 12 QSO/galaxy SED pairs (3 QSO times 4 galaxy SEDs) we vary the normalisation of the QSO component relative to the galaxy component between 0.1 and 0.9 in steps of 0.2 , and we then sum the weighted SED pairs. This results in 5 linear combinations for each QSO/galaxy SED pair and therefore a total of 60 combined QSO/galaxy templates. We find that using these 60 templates does not significantly improve the photometric redshift estimates of optically extended blue $(g-r<0.5)$ objects: only two additional sources are assigned photometric redshifts accurate within $\delta z \lesssim 0.15$.

Finally, for the optically extended spectroscopic subsample there is only 1 source with $r>21 \mathrm{mag}$, and therefore we cannot comment on the success rate of our method for fainter sources where the star/galaxy separation becomes less reliable. For this one source (\#10 in Table 1) the photometric 


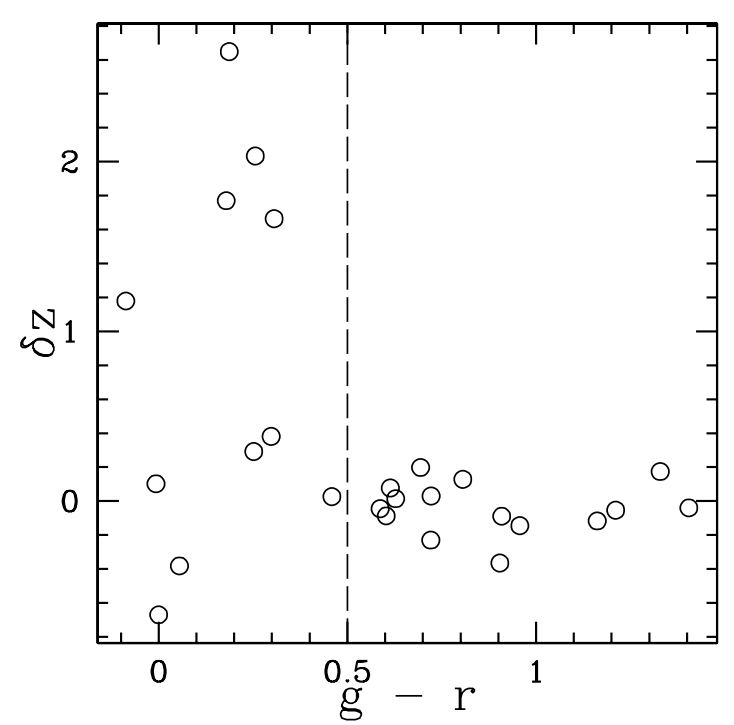

Fig. 2. $\delta z$ against $g-r$ colour for optically extended X-ray sources with spectroscopic redshift measurements. Our method provides reasonable photometric redshift estimates for relatively red sources $(g-r>$ $0.5)$. For bluer optical colours the accuracy of our method is poorer, most likely because of contamination of the optical light from the central engine.

redshift estimate is not in good agreement with the spectroscopic redshift measurement, but this is most likely because of the blue colour of the object.

\subsection{The spectroscopically unidentified sub-sample}

In this section we apply the HMP00 method to X-ray sources for which no spectroscopic data are available. This sub-sample comprises 96 sources of which 45 have point-like optical morphology and 51 are optically extended. From the point-like subsample we exclude one source with $\log \left(f_{\mathrm{X}} / f_{\mathrm{opt}}\right)<-1$ that is also best fit with a stellar template. Based on the discussion in Sect. 4.1, this is likely to be a Galactic star. The results for the remaining 44 sources are presented in Table 2 . We also caution the reader that there are 24 point-like $\mathrm{X}$-ray sources with $r>21 \mathrm{mag}$ for which, as discussed above, our method is expected to perform less well.

The results for the 51 optically extended sources in the spectroscopically unidentified sub-sample are also presented in Table 2. 29 of these sources have red optical colours $(g-r>0.5 \mathrm{mag})$ for which our method gives acceptable photometric redshift estimates. The remaining 22 sources however, have $g-r<0.5 \mathrm{mag}$ and as discussed above their photometric redshifts are not reliable. Nevertheless, for the sake of completeness these are also listed in Table 2.

\section{Discussion}

In this paper we describe a recipe for estimating photometric redshifts for bright X-ray selected samples comprising both powerful distant QSOs and less luminous lower-Z Seyfert-type systems. Firstly, we exclude from our analysis $\mathrm{X}$-ray sources associated with Galactic stars by identifying

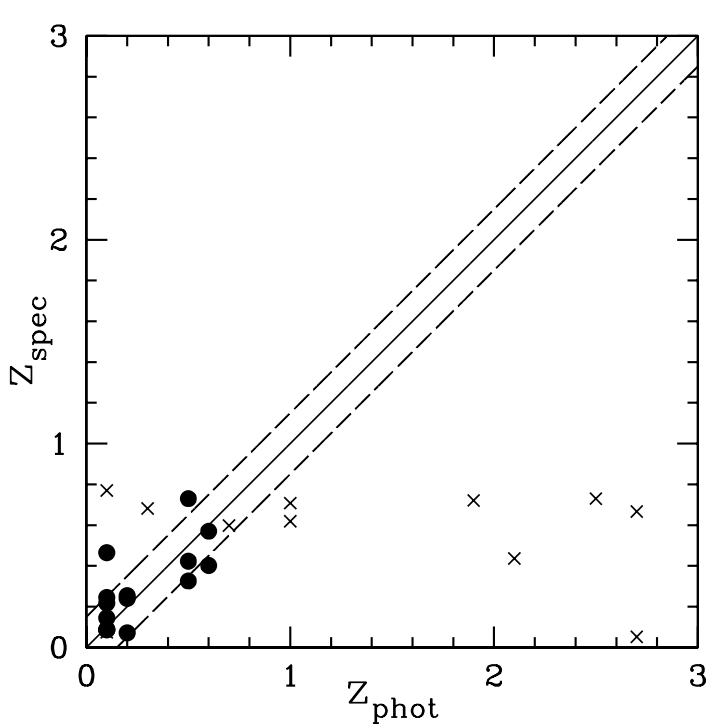

Fig. 3. Comparison between $z_{\text {spec }}$ and $z_{\text {phot }}$ for X-ray sources with extended optical light profile and spectroscopic redshift measurements. Filled circles are for red $(g-r>0.5)$ and crosses are for blue $(g-r<0.5)$ sources. The solid line is the $z_{\text {spec }}=z_{\text {phot }}$ locus. The dashed lines define the $\delta z= \pm 0.15$ envelope around the $z_{\text {spec }}=z_{\text {phot }}$ line.

optically unresolved objects with low X-ray-to-optical flux ratio $\left(\log f_{\mathrm{X}} / f_{\mathrm{opt}}<-1\right)$ and SEDs that are best fit with stellar templates. The remaining X-ray sources are split into optically point-like and extended systems. The former are most likely associated with powerful QSOs where the central engine dominates the optical light. The latter are low-luminosity AGNs at moderate redshifts and therefore their optical colours also have a significant stellar component from the host galaxy. For optically point-like objects we use QSO templates to estimate their photometric redshifts while for optically extended X-ray sources we use galaxy SEDs.

This method is applied to all the spectroscopically identified X-ray sources detected in the present XMM-Newton/2dF sample. For optically extended sources with relatively red colours $(g-r>0.5)$ the photometric redshift success rate is reasonably good: i) $\sim 73$ per cent have $\delta z \leq 0.15$; ii) the fraction of non-catastrophic redshifts, i.e. with $\delta z /(1+z) \leq 0.2$, is 93 per cent; iii) the rms scatter is $\sigma_{z}=0.10$. The fraction of problematic photometric redshift estimates increases, however, for optically extended objects with $g-r<0.5$, most likely because of contamination of their optical light by emission from the central engine. For these sources we find that 27 per cent have $\delta z /(1+z) \leq 0.2$ and we estimate an rms scatter of $\sigma_{z}=0.99$. We find no improvement when QSO or star-forming galaxy templates, or linear combinations of QSO and galaxy SEDs, are applied to these systems. In addition the application of a distance prior $(z<1)$ results in no significant improvement of the results, with the rms scatter still large, i.e. $\sigma_{z}=0.25$.

For point-like sources we estimate an accuracy of $\delta z \lesssim$ 0.3 for $\approx 68$ per cent of the sample. About 75 per cent of these sources have $\delta z /(1+z) \lesssim 0.2$. Figure 1 shows that there are degeneracies that affect the efficiency of our method, 
Table 2. Photometric redshifts for the 95 X-ray sources without spectroscopic identification in the XMM-Newton/2dF sample.

\begin{tabular}{|c|c|c|c|c|c|c|c|c|c|c|}
\hline \multirow[t]{2}{*}{$\#$} & \multicolumn{3}{|c|}{$\overline{\alpha_{\text {opt }}(\mathrm{J} 2000)}$} & \multicolumn{3}{|c|}{$\overline{\delta_{\text {opt }}(\mathrm{J} 2000)}$} & \multirow[t]{2}{*}{$z_{\text {phot }}$} & \multirow[t]{2}{*}{$r^{1}$} & \multirow[t]{2}{*}{$g-r^{1}$} & \multirow[t]{2}{*}{ Morph } \\
\hline & $\mathrm{h}$ & $\mathrm{m}$ & $\mathrm{s}$ & $\bar{o}$ & ' & " & & & & \\
\hline 1 & 13 & 40 & 38.61 & 00 & 19 & 19.52 & 0.500 & 19.626 & 0.770 & $\mathrm{E}$ \\
\hline 2 & 13 & 40 & 43.05 & 00 & 17 & 03.70 & 3.100 & 22.048 & 0.154 & $\mathrm{E}$ \\
\hline 3 & 13 & 40 & 44.02 & 00 & 27 & 03.96 & 4.280 & 20.253 & 1.235 & $\mathrm{P}$ \\
\hline 4 & 13 & 40 & 44.89 & 00 & 26 & 18.13 & 2.140 & 21.990 & -0.093 & $\mathrm{P}$ \\
\hline 5 & 13 & 40 & 44.99 & -00 & 24 & 03.92 & 0.500 & 20.185 & 1.510 & $\mathrm{E}$ \\
\hline 6 & 13 & 40 & 52.49 & 00 & 20 & 06.76 & 0.800 & 21.524 & 0.459 & $\mathrm{E}$ \\
\hline 7 & 13 & 40 & 58.59 & -00 & 22 & 33.38 & 3.300 & 18.319 & 0.703 & $\mathrm{P}$ \\
\hline 8 & 13 & 40 & 59.90 & -00 & 34 & 08.04 & 1.600 & 21.914 & 0.209 & $\mathrm{P}$ \\
\hline 9 & 13 & 41 & 14.44 & 00 & 20 & 31.02 & 3.000 & 21.168 & 0.982 & $\mathrm{E}$ \\
\hline 10 & 13 & 41 & 18.35 & 00 & 26 & 41.10 & 1.700 & 21.782 & 0.087 & $\mathrm{P}$ \\
\hline 86 & 13 & 45 & 07.47 & 00 & 04 & 06.82 & 0.500 & 19.529 & 0.842 & E \\
\hline 87 & 13 & 45 & 08.36 & 00 & 22 & 27.98 & 0.100 & 21.055 & 1.017 & $\mathrm{E}$ \\
\hline 88 & 13 & 45 & 09.64 & 00 & 20 & 52.80 & 0.500 & 19.542 & 1.338 & $\mathrm{E}$ \\
\hline 89 & 13 & 45 & 09.80 & -00 & 27 & 40.75 & 1.500 & 20.228 & 0.620 & $\mathrm{P}$ \\
\hline 90 & 13 & 45 & 10.33 & 00 & 18 & 52.31 & 0.200 & 19.174 & 0.797 & $\mathrm{E}$ \\
\hline 91 & 13 & 45 & 12.53 & -00 & 17 & 45.17 & 2.140 & 21.443 & 0.194 & $\mathrm{P}$ \\
\hline 92 & 13 & 45 & 15.77 & 00 & 28 & 51.06 & 0.900 & 21.292 & 0.351 & $\mathrm{E}$ \\
\hline 93 & 13 & 45 & 15.88 & -00 & 17 & 53.81 & 2.700 & 21.218 & 0.330 & $\mathrm{E}$ \\
\hline 94 & 13 & 45 & 19.85 & 00 & 28 & 50.66 & 0.700 & 20.244 & 0.362 & $\mathrm{E}$ \\
\hline 95 & 13 & 45 & 22.59 & 00 & 01 & 00.19 & 0.400 & 20.940 & 0.422 & $\mathrm{E}$ \\
\hline
\end{tabular}

${ }^{1} r$ : SDSS $r$ band magnitude; $g-r$ : SDSS $g-r$ colour in mag; morph: SDSS optical morphology (P - point-like; E - extended).

particularly for sources with $z \gtrsim 1.5$. As discussed by Richards et al. (2001), this is likely because of strong emission lines falling between the SDSS filters at certain redshift ranges. Indeed, these authors use a different method to that described here to estimate photometric redshifts for optically selected SDSS QSOs. They find that photometric redshifts for 70 per cent of the sources in their sample are correct within $\delta z<0.2$ with an rms scatter of 0.67 , estimated on their whole sample ( $\sim 2600$ sources). Their success rate for optically selected QSOs is better than the one obtained here for $X$-ray selected QSOs: 52 per cent have $\delta z<0.2$ and the rms scatter is estimated to be 0.72 (based on our sample of 62 sources) ${ }^{1}$. Nevertheless, similarly to our results, the accuracy of their method is also lower for sources in the range $1.5 \lessgtr z \lesssim 2.5$. They attribute these problems to the MgII, CIV and Ly $\alpha$ lines falling between the $u$ and $g$ bands at different redshifts.

We also explore the possibility that the colours of our X-ray selected AGNs are responsible for the problematic redshifts at $z \gtrsim 1.5$. Richards et al. (2001) found that about 4 per cent of their optically selected AGN sample have colours redder than those of typical QSOs at $z \lesssim 2.2$ (i.e. $u-g \gtrsim 0.6$ ) suggesting

\footnotetext{
1 We note that our $X$-ray selected spectroscopic sub-sample does not identify sources at redshifts larger than 3 , a redshift range where Richards et al. (2001; see their Fig. 2) have an increased success rate. We also expect our method to perform similarly well at this redshift range (HMP00).
}

either reddening or high-z systems. A large fraction of these redder sources has inaccurate photometric redshift estimates. Since X-ray selection is least biased to obscured AGNs we also expect a non-negligible fraction of QSOs with red colours most likely because of dust extinction. Estimating the redshift of these systems is problematic since their SEDs are likely to be very different to the templates used by the HMP00 method.

Figure 4 plots $u-g$ against $g-r$ for all the X-ray selected sources with unresolved optical morphology. The curves are the colour-colour tracks of the HMP00 QSO templates in the redshift range $0-6$ for two different values of the optical powerlaw spectral index, 0 and 1 (solid and long-dashed line respectively). The $z \gtrsim 2$ regime for these models lies at $u-g \gtrsim 0.6$. A number of sources are redder than $u-g>0.6$ (total of 21) but these are most likely high-z rather than dusty QSOs. Indeed, all sources with spectroscopic identification and $u-g \gtrsim 0.6$ have $z_{\text {spec }} \gtrsim 2$. Nevertheless, a striking result in Fig. 4 is the nonnegligible fraction of optically point-like $\mathrm{X}$-ray sources that are red in both the $u-g$ and the $g-r$ colours (7 per cent with $u-g>0.6$ and $g-r>0.6$ ). These red colours cannot be accounted for by the HMP00 QSO templates at any redshift and suggest the presence of dust. Unfortunately spectroscopic redshifts are not available for these sources to explore their nature. It is likely however, that the photometric redshifts for these systems are problematic since their colours are much redder than 


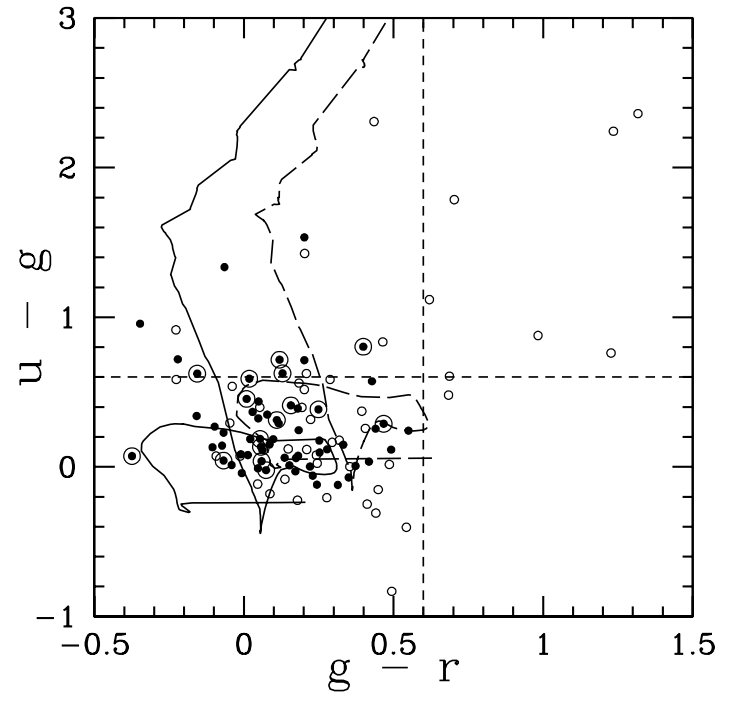

Fig. 4. Colour-colour diagram of the X-ray sources with point-like optical morphology. Open circles are for sources without $z_{\text {spec }}$, filled circles correspond to sources in the spectroscopic sub-sample. A large circle on top of a symbol is for sources with problematic photometric redshift estimates $(\delta z>0.3)$. The two curves are colour-colour tracks of the HMP00 QSO templates in the redshift range 0-6 for two different values of the optical power-law spectral index, 0 and 1 for the solid and the long-dashed lines respectively. For $u-g \gtrsim 0.6$ these templates correspond to $z \gtrsim 2$. The short-dashed horizontal and vertical lines correspond to $u-g=0.6$ and $g-r=0.6 .7$ point-like X-ray sources have colours redder than these limits. The photometric redshifts of these sources are likely to be problematic since they may have SEDs substantially different to those expected for optically selected QSOs.

those of optically selected QSOs at any redshift. A different set of template SEDs is probably required for such sources.

Babbedge et al. (2004) recently presented a method similar to the one described here to estimate photometric redshifts for both QSOs and galaxies in the ELAIS N1 and N2 fields as well as in the Chandra Deep Field North region. The additional criteria applied by our method are the use of the X-ray to optical flux ratios to identify stars and the separation between blue and red galaxies. The application of these is essential in bright X-ray samples which comprise an elevated fraction of both stars and sources having blue colours. The accuracy of the photometric redshift estimates of Babbedge et al., in the case of the CDFN region, is in reasonable agreement with those obtained here. The above authors find that 83 per cent of their QSO sample have $\delta z /(1+z)<0.2$; in our case this fraction amounts to 75 per cent. For the galaxies Babbedge et al. estimate that a fraction of 90 per cent has $\delta z /(1+z)<0.2$, while we find a fraction of 93 per cent for the red galaxies only. We find however that there are limitations in the application of photometric redshifts to the blue optically resolved sources.

\section{Conclusions}

In this paper we explore the use of photometric redshifts to bright X-ray samples. In particular, we estimate photometric redshifts for a sample of 193 X-ray sources with optical counterparts in the XMM-Newton/2dF survey. This is a bright
X-ray sample $\left[f_{\mathrm{X}}(0.5-8 \mathrm{keV}) \approx 10^{-14} \mathrm{erg} \mathrm{s}^{-1} \mathrm{~cm}^{-2}\right]$ consisting of both powerful distant QSOs and lower luminosity nearby AGNs. We split our sample into extended and pointlike sources based on their optical light profile. We then employ the HMP00 technique to determine photometric redshifts, using galaxy and QSO templates for the extended and the pointlike sources respectively. A number of stars can be immediately identified on the basis of their unresolved optical light profile, their low X-ray-to-optical flux ratio and their SEDs that are best fit with stellar templates.

Comparison between photometric and spectroscopic redshifts for 62 optically unresolved sources shows that for $\approx 68$ per cent the photometric redshift estimates are accurate within $\delta z \lessgtr 0.3$. The photometric redshifts appear to be in good agreement with spectroscopic ones up to $z \approx 1.5$. At larger redshifts the presence of emission lines between filters limits the efficiency of our method. We also find a non-negligible fraction $(7 \%)$ of X-ray sources with point-like optical profiles that have colours redder than those of optically selected QSOs. The photometric redshift estimates for these sources are likely to be problematic.

For X-ray sources with extended optical light profiles we find that galaxy templates provide reasonable ( 73 per cent have $\delta z<0.15)$ photometric redshifts at least for sources with red colours $(g-r>0.5)$. We find however that a combined galaxyQSO template approach cannot be successfully applied to optically extended sources with bluer colours. These present poor photometric redshift estimates, regardless of whether the estimates are based on galaxy or QSO templates, or any linear combination of the two.

Acknowledgements. We thank the anonymous referee for valuable comments and suggestions. This work is funded by the European Union and the Greek Ministry of Development in the framework of the Programme "Competitiveness-Promotion of Excellence in Technological Development and ResearchAction 3.3.1", Project "X-ray Astrophysics with ESA's mission XMM", MIS-64564. The XMM-Newton/2dF survey data as well as part of the observations presented here are electronically available at http://www.astro.noa.gr/xray/.

The $2 \mathrm{dF}$ QSO Redshift Survey (2QZ) was compiled by the 2QZ survey team from observations made with the 2-degree Field on the Anglo-Australian Telescope.

Funding for the creation and distribution of the SDSS Archive has been provided by the Alfred P. Sloan Foundation, the Participating Institutions, the National Aeronautics and Space Administration, the National Science Foundation, the US Department of Energy, the Japanese Monbukagakusho, and the Max Planck Society. The SDSS Web site is http://www.sdss.org/. The SDSS is managed by the Astrophysical Research Consortium (ARC) for the Participating Institutions. The Participating Institutions are The University of Chicago, Fermilab, the Institute for Advanced Study, the Japan Participation Group, The Johns Hopkins University, Los Alamos National Laboratory, the Max-Planck-Institute for Astronomy (MPIA), the Max-Planck-Institute for Astrophysics (MPA), New Mexico State University, University of Pittsburgh, Princeton University, the United States Naval Observatory, and the University of Washington. 


\section{References}

Babbedge, T. S. R., Rowan-Robinson, M., Gonzalez-Solares, E., et al. 2004, MNRAS, 353, 654

Barcons, X., Carrera, F. J., Watson, M. G., et al. 2002, A\&A, 382, 522 Barger, A. J., Cowie, L. L., Brandt, W. N., et al. 2002, AJ, 124, 1839

Brandt, W. N., Alexander, D. M., Hornschemeier, A. E., et al. 2001, AJ, 122, 2810

Brandt, W. N., Schneider, D. P., Fan, X., et al. 2002, ApJ, 569, L5

Budavári, T., Csabai, I., Szalay, A. S., et al. 2001, AJ, 122, 1163

Budavári, T., Connolly, A. J., Szalay, A. S., et al. 2003, ApJ, 595, 59

Coleman, G. D., Wu, C.-C., \& Weedman, D. W. 1980, ApJ, 43, 393

Croom, S. M., Smith, R. J., Boyle, B. J., et al. 2001, MNRAS, 322, 29

Csabai, I., Budavári, T., Connolly, A. J., et al. 2003, AJ, 125, 580

Downes, A. J. B., Peacock, J. A., Savage, A., \& Carrie, D. R. 1986, MNRAS, 218, 31

Fukugita, M., Ichikawa, T., Gunn, J. E., et al. 1996, AJ, 111, 1748

Georgakakis, A., Georgantopoulos, I., Stewart, G. C., Shanks, T., \& Boyle, B. J. 2003, MNRAS, 344, 161

Georgakakis, A., Georgantopoulos, I., Vallbé, M., et al. 2004, MNRAS, 349, 135

Georgantopoulos, I., Stewart, G. C., Shanks, T., Boyle, B. J., \& Griffiths, R. E. 1996, MNRAS, 280, 276
Georgantopoulos, I., Georgakakis, A., Akylas, A., et al. 2004, MNRAS, 352, 91

Giacconi, R., Rosati, P., Tozzi, P., et al. 2001, ApJ, 551, 624

Gonzalez, A. H., \& Maccarone, T. J. 2002, ApJ, 581, 155

Green, P. J., Silverman, J. D., Cameron, R. A., et al. 2004, ApJS, 150, 43

Hasinger, G., Altieri, B., Arnaud, M., et al. 2001, A\&A, 365, L45

Hatziminaoglou, E., Mathez, G., \& Pelló, R. 2000, A\&A, 359, 9

Hatziminaoglou, E., Groenewegen, M. A. T., da Costa, L., et al. 2002, A\&A, 384, 81

Kim, D.-W., Cameron, R. A., Drake, J. J., et al. 2004a, ApJS, 150, 19

Kim, D.-W., Wilkes, B. J., Green, P. J., et al. 2004b, ApJ, 600, 59

Madau, P. 1995, ApJ, 441, 18

Mainieri, V., Bergeron, J., Hasinger, G., et al. 2002, A\&A, 393, 425

Mobasher, B., Idzi, R., Benítez, N., et al. 2004, ApJ, 600, L167

Pickles, A. J. 1998, MNRAS, 110, 863

Richards, G. T., Weinstein, M. A., Schneider, D. P., et al. 2001, AJ, 122,1151

Richards, G. T., Fan, X., Newberg, H. J., et al. 2002, AJ, 123, 2945

Stocke, J. T., Morris, S. L., Gioia, I. M., et al. 1991, ApJS, 76, 813

Stoughton, C., Lupton, R. H., Bernardi, M., et al. 2002, AJ, 123, 485

Wang, T. G., Lu, Y. J., \& Zhou, Y. Y. 1998, ApJ, 493, 1

York, D. G., Adelman, J., Anderson, J. E. Jr., et al. 2000, AJ, 120, 1579 